\title{
Promoting co-production in the generation and use of research evidence to improve service provision in special care dentistry
}

\author{
Paul R. Brocklehurst, ${ }^{* 1}$ Joe Langley, ${ }^{2}$ Sarah R. Baker, ${ }^{3}$ Gerald McKenna, ${ }_{1}^{4}$ Craig Smith ${ }^{5}$ and Rebecca Wassall ${ }^{6}$
}

\section{Key points}

Involving non-researchers (patients, professionals, policy makers) in any or all stages of research will result in relevant research, contextually sensitive interventions and meaningful measures.
In fields such as special care dentistry that require tailored approaches to patient care, co-produced research is arguably very relevant.
Involving non-researchers requires a genuine sharing of power in the research process. It is important not to under-estimate the level of expertise and skill required to deliver the facilitative methods used to in co-production.

\begin{abstract}
Special care dentistry (SCD) provides holistic oral service provision for people with complex health and care needs. These can include physical, sensory, intellectual, mental, medical, emotional or social impairment or disability or, more often, a combination of these factors. The level of disability within these population groups can vary, and a proportion of people will have multiple and overlapping impairments and/or medical conditions. This paper explores a number of possible research methods that may better reflect the diversity and challenges of this population group, where the emphasis is placed on co-production and co-design.
\end{abstract}

\section{Introduction}

Special care dentistry (SCD) provides holistic oral service provision for people with complex health and care needs. ${ }^{1}$ These can include physical, sensory, intellectual, mental, medical, emotional or social impairment or disability or, more often, a combination of these factors. As a result, the remit of SCD is broad and covers a heterogeneous population group. The level of disability within these population groups can also vary, and a proportion of people will have multiple and overlapping impairments and/or medical conditions. ${ }^{2}$ From a clinical perspective, it can often require 'a holistic

'Director of NWORTH Clinical Trials Unit and Honorary Consultant in Dental Public Health, Bangor University, The Normal Site, Holyhead Road, Gwynedd, UK; 2Sheffield Hallam University, Art and Design Research Centre, Cantor Building, Sheffield, UK; ${ }^{3}$ University of Sheffield, School of Clinical Dentistry, Sheffield, UK; ' ${ }^{Q}$ Queens University Belfast, Nutrition and Metabolism Group, Centre for Public Health, Belfast, UK; ${ }^{5}$ University of Manchester, School of Biological Sciences, Manchester, UK; ' $U$ University of Newcastle, School of Dental Sciences, Newcastle, UK.

Correspondence to: Paul Brocklehurst

Email: p.brocklehurst@bangor.ac.uk

Accepted 19 November 2018

DOI: 10.1038/s41415-019-0458-5 approach that is specialist-led in order to meet the complex requirements of people with impairments. ${ }^{3}$ It can also require a highly tailored approach, where the individual's clinical needs are carefully considered alongside the patient's expressed and wider medical, health and care needs.

Given the importance of these elements, it could be argued that it requires a more considered approach to the generation and use of research evidence. This is challenging as the academic literature for this heterogeneous population group is not as mature as it is for those without disability and impairment. Over the last ten years, very few large-scale trials or studies have been undertaken in SCD. ${ }^{4}$ Currently, there is only one trial that is looking at the effect of a high fluoride toothpaste in a potentially relevant population group, but this is likely to only form a small proportion of those that will be eventually recruited. ${ }^{5}$ Equally, the role of context in oral care for people living with disability and impairment is key. An intervention or approach that might work with one patient may not work with another, given the need to account for other personal, clinical, emotional, medical and environmental factors. This poses a challenge, as we know in the broader literature that there is generally 'not enough contextual information provided to transfer the results from the trial setting into other settings. ${ }^{6}$ The role of context can also be stripped out further by the process of evidence synthesis. As Northridge and Metcalf highlight, there is a 'need to extract the core issues from the context in which they are embedded in order to better ensure that they are transferable across settings.7 As a result, this paper explores a number of possible research methods that may better reflect the diversity and challenges of this population group, where the emphasis is placed on co-production and co-design; that is, where research is carried out with evidenceusers 'rather than to, about, or for them.'

\section{Importance of co-production}

Understanding evidence-users' needs and the challenges of improving health and wellbeing is important. ${ }^{9}$ Greenhalgh et al. argue that the best way to ensure that evidence is used is to co-create knowledge, drawing on the principles of co-production. ${ }^{10}$ As Langley et al. highlight, co-production in this sense adopts an inductive paradigm of partnership working, positioning research as a creative enterprise 
that has human experience at its core, while paying attention to the quality of relationships within the resulting partnership. ${ }^{10,11}$ Using facilitative methods, this approach is argued to bridge the gap between knowledge producers and knowledge users, and would appear to offer some promise for SCD. ${ }^{12}$

Inherently, co-production and co-creation challenges predetermined, structural and often unstated assumptions around power and helps to ameliorate epistemological injustice; that is, it challenges just who is allowed to control the knowledge agenda. ${ }^{13}$ As Langley et al. highlights, each stakeholder group (for example, people with disability and/or impairment, clinicians, commissioners and researchers) will bring 'different cognitive and emotional representation on [an] issue, shaped by different experiences and interests. ${ }^{14}$ As a result:

'a shared understanding of the nature of research and potential contributions to the research process has [....] to be considered within a dynamic context of different stakeholders' mental models, which can be used to deconstruct and advance the knowledge problem towards potential solutions..$^{14,15}$

This paper will discuss three approaches to co-production in three key areas: 1) developing the research agenda; 2) developing the intervention; and 3) developing measures for evaluation. Each will be detailed briefly to elaborate on an earlier paper, using examples from ongoing work in the area. ${ }^{16}$

\section{Developing the research agenda}

Priority setting partnerships (PSPs) are based on a consensus methodology and were developed initially by the James Lind Alliance to determine the most pressing research issues for any given population group. More specifically, they 'promote discussion about how patients, clinicians and policymakers should respond to uncertainties about the effects of treatments. ${ }^{17,18}$ PSPs use a modified 'nominal group technique' which builds consensus across a range of stakeholders, ensuring the narratives of knowledge users (patients, clinicians and commissioners) are heard alongside those of knowledge producers (researchers). ${ }^{19}$ As such, they help to ensure that research agendas are built on the needs of the former, rather than being dictated by the latter.

One example of this approach is the ongoing PSP being undertaken by the National Institute for Health Research (NIHR) speciality leads in oral health across the United Kingdom. ${ }^{20}$ The aim of this PSP is to 'identify the unanswered questions related to oral and dental health from patient and clinical perspectives and then prioritise those that patients and clinicians agree are the most important'. One extension of this approach would be to run a PSP specifically for the population groups cared for by SCD, as the careful and thoughtful use of creative approaches can allow people (like many patients in SCD) who are normally excluded from such activities to be heard. A similar approach for dependent older people was piloted in 2015 in both the UK and the Netherlands. ${ }^{21,22}$ A summary of these two PSPs has recently been reported and the outcomes framed using Maxwell's taxonomy on quality. ${ }^{23}$ Key stakeholders were asked to explore a series of stem questions for discussion and present their views, which were discussed in four separate groups (users of services, carers of users of services, clinicians and care home staff). A shared ranking exercise was then undertaken after further structured small group discussions. Based on the nominal group technique, each group took part in a facilitated discussion to identify key local priorities, which are provided in detail in the three published papers. ${ }^{21,22,23}$

\section{Developing the intervention}

As highlighted by Langley, 'design is both a practice and a process. ${ }^{11}$ Design helps to make ideas tangible, develop practical and attractive propositions to evidence-users and is particularly suited to complex, ill-defined problems, involving stakeholders with different perspectives. ${ }^{24,25} \mathrm{Co}$-design has an emphasis on process, where facilitation and co-creation brings different participants together to elicit and share their experience and perspectives. ${ }^{26}$ Co-design recognises that stakeholders can bring both explicit and tacit knowledge and that working together in a group can help surface the latter and create new shared meaning that remains visible to all stakeholders throughout the process (given the 'ongoing physical presence of the prototypes'). ${ }^{11}$

One relevant example here is the use of 'experience-based co-design' (EBCD). In a NIHR-funded study, researchers from Manchester, Bangor and Northumbria universities are developing a 'stroke friendly oral health promoting' (STOP) toolkit to improve oral self-care practices after discharge from hospital stroke services. Dental disease is highly prevalent in people with stroke and there is growing evidence of a potential shared inflammatory pathway. ${ }^{27}$ People who have suffered from a stroke have higher levels of both dental caries and periodontal disease and common risk factors such as smoking. ${ }^{28}$ Stroke survivors tend to have fewer teeth compared to the rest of the population, and often wear dentures. ${ }^{29}$ Xerostomia is common due to stroke-related medication, which can further significantly increase the risk of tooth decay, periodontal disease, oral infection (for example, oral thrush) and impact negatively upon wearing dentures. ${ }^{30}$ In turn, poor oral health has been linked with important sequelae of stroke such as aspiration pneumonia, reduced quality of life and poor nutritional status. ${ }^{31}$

EBCD is an approach that puts users at the centre of the design process by first capturing their experiences of care and then uses summaries of these experiences to develop new interventions or pathways. ${ }^{32,33}$ In the STOP toolkit study, researchers are first using qualitative interviews to understand the dental care experiences of stroke survivors, how they manage oral self-care practices, the context of the proposed intervention and what 'ideal' would look like. These experiences are being captured on video and then a trigger film will be created to relay 'touch-points' (points in the interview that are imbued with affect or that have an identified 'key-ness'), in readiness for the design stage.

At the design stage, evidence-users (stroke sufferers, their carers, clinicians and commissioners) and evidence-producers will be brought together in four stages to develop the toolkit. The facilitators of the EBCD workshops will collate the expressed needs of stroke patients, along with their preferences and contributions. These will be represented on wall-charts and flipcharts to co-create, in real time, a thematically organised map of the group's thoughts, including important areas to aid in the development of the toolkit. The information will then be photodocumented and used to inform the toolkit's content (for example, education, information provision, signposting) and its format (design, layout, accessibility and availability). These will then be based on APEASE criteria (affordability, practicability, effectiveness and cost-effectiveness, acceptability, side-effects/safety and equity). ${ }^{34}$

\section{Developing measures for evaluation}

Using co-production to develop the research agenda and to design new interventions are two important areas that are of potential relevance for SCD. Another important area where co-production is important is in determining the types of outcome measures 
that are used when we evaluate the effectiveness of interventions, based on an experimental or quasi-experimental design. As highlighted by Kirkham et al., there is:

'[a] growing recognition that insufficient attention has been paid to the outcomes measured in clinical trials, which need to be relevant to health service users and other people making choices about health care if the findings of research are to influence practice and future research., ${ }^{35}$

As a result, core outcome sets, which account for the views of evidence-users are increasingly being recognised as an important step forward. Recent standards have been published to guide the development of COSs (consensusbased standards) for the selection of health measurement instruments (COSMIN); and core outcome set standards for reporting (COS-STAR) statement). ${ }^{33,36}$ The COMET (core outcome measures in effectiveness trials) initiative brings together people interested in the development and application of agreed standardised sets of outcomes, and holds an online database of planned, ongoing and completed work.

One example here, that is relevant for SCD, is the study being run by researchers from Queens University Belfast, alongside Bangor, Glasgow and Newcastle universities and University College London: 'DEvelopment of a Core outcome set for orAl health services research involving DEpendent older adults (DECADE) ${ }^{37}$ This study will build on the PSP identified above, which was used to set the research agenda, alongside an effectiveness practice and organisation of care Cochrane review. ${ }^{38}$ These will be used to develop an initial set of opening questions for qualitative interviews with dependent older people, their carers, care home staff, clinicians and commissioners of NHS services. ${ }^{20}$ The interviews will then be transcribed and undergo thematic analysis. At the consensus stage, the different stakeholders will be asked to score each outcome from a long list of identified outcome measures gleaned from the systematic review and the previous stages of the process. This will be undertaken in stages, similar to processes already utilised in dentistry. ${ }^{39}$ Subsequent approaches for the final selection of the COS include the scale proposed by grade: 1-3 signifies an outcome of limited importance, 4-6 important but not critical, and 7-9 critical.

A number of rounds across multiple stakeholder groups will be held, using the grade criteria. This will enable the research team to summarise the responses and feed this back to the stakeholder groups to produce a refined version. To be consistent with the approach, an outcome will be included in the COS if more than $70 \%$ of the stakeholders score the measure between 7 and 9 and if fewer than $15 \%$ of the stakeholders score it as 1 to 3. Equally, consensus that an outcome is not included in the COS will be defined as $70 \%$ or more scoring it as 1 to 3 and fewer than $15 \%$ scoring it as 7 to $9 .{ }^{40}$

\section{Summary}

Given the complex requirements of people with disability and impairments, and the holistic and tailored approach to clinical management that is commonly necessary, it would appear that co-production has much to offer SCD. Three brief examples have been provided that outline how such an approach may help in the generation and use of research evidence. All adopt an inductive paradigm of partnership working, positioning research as a creative enterprise that has human experience at its core. Using facilitative methods in the development of research agendas, intervention development and outcome measurement helps to narrow the gap between knowledge producers and knowledge users, while heralding an approach that ensures that the experiences and knowledge of all stakeholders are considered equally.

In the first example, issues around power were addressed by using different stakeholder groups to set the initial priorities at separate meetings. These were then refined at a final meeting which contained representatives of each of these groups, but chaired by a patient representative to ensure the views of users of services were given weight. In the second example, the design of the intervention will again be considered by individual groups during the first iteration of the toolkit, with stroke patients themselves driving the notemaking and collation process. Clinicians and commissioners of care will input into the design stage in separate groups undertaken concurrently, but again, the pooling of the different ideas from the different groups will be steered by stroke patient representatives in the final amalgamated group and the subsequent meetings. The use of patients in the development of COSs is now fully recognised and the explanatory document accompanying the COS-STAR statement has multiple references to end users of services to ensure that their views are represented. ${ }^{35}$ In rheumatology research, the explicit inclusion of patients in the development of COS has 'significantly influenced outcome research in the field... identifying new domains that are important for patients, and provided the patient perspective. ${ }^{41}$ Overall, this has 'led to wider patient involvement as partners in research' and now is being used in $81 \%$ of trials on the ClinicalTrials.gov database, two pertinent goals for research in SCD. ${ }^{41,42}$

\section{References}

1. World Health Organisation. International Classification of Functioning, Disability and Health. 2018. Available at https://www.who.int/classifications/icf/en/ (accessed May 2019)

2. Gallagher J E, Fiske J. Special Care Dentistry: a professional challenge. Br Dent J 2007; 202: 619-629.

3. Royal College of Surgeons. Specialty Training Curriculum: Special Care Dentistry. 2009. Available at https://www. rcseng.ac.uk/-/media/files/rcs/fds/publications/curricula/ scdcurriculumfebruary2010.pdf (accessed May 2019).

4. National Institute for Health Research. Journals Library. Available at https://www.journalslibrary.nihr.ac.uk/\#/ (accessed May 2019).

5. University of Aberdeen. REFLECT trial. Available at https://w3.abdn.ac.uk/hsru/REFLECT/Public/Public/ index.cshtml (accessed May 2019).

6. Grant A, Treweek S, Wells M. Why is so much clinical research ignored and what can we do about it? $\mathrm{Br} J$ Hosp Med (Lond) 2016; 77 (Spec Iss): 554-555.

7. Northridge M E, Metcalf S S. Enhancing implementation science by applying best principles of systems science. Health Res Policy Syst 2016; 14: 74.

8. The Involve Foundation. Available at https://www. involve.org.uk/ (accessed May 2019).

9. Luxford K, Safran D G, Delbanco T. Promoting patientcentered care: a qualitative study of facilitators and barriers in healthcare organizations with a reputation for improving the patient experience. Int I Qual Health Care 2011; 23: 510-515.

10. Greenhalgh T, Jackson C, Shaw S, Janamina T. Achieving research impact through co-creation in communitybased health services: literature review and case study. Millbank Q 2016; 94: 392-429.

11. Langley J, Wolstenholme D, Cooke J. Collective making as knowledge mobilisation: the contribution of participatory design in the co-creation of knowledge in healthcare. BMC Health Serv Res 2018; 18: 585.

12. Rycroft-Malone J, Burton C R, Bucknall T, Graham I D, Hutchinson A M, Stacey D. Collaboration and Co-Production of Knowledge in Healthcare: Opportunities and Challenges. Int J Health Policy Manag 2016; 5: 221-223.

13. Fricker M. Epistemic Injustice: Power and the Ethics of Knowing. Oxford: Oxford University Press, 2007.

14. Rycroft-Malone J, Burton C, Wilkinson J et al. Collective action for knowledge mobilisation: a realist evaluation of the collaborations for leadership in applied health research and care. Health Serv Deliv Res 2015; DOI: 10.3310/hsdr03440

15. Ryan B. Co-production: Option or Obligation? Aust J Publ Admin 2012; 71: 314-324

16. Brocklehurst P R, McKenna G, Schimmel M et al. How do we incorporate patient views into the design of healthcare services for older people: a discussion paper. BMC Oral Health 2018; 18: 61.

17. Chalmers I, Atkinson P, Fenton M, Firkins L, Crowe $S$, Cowan $\mathrm{K}$. Tackling treatment uncertainties together: the evolution of the James Lind initiative 2003-2013. J R SoC Med 2013; 106: 482-491.

18. Abma T A, Nierse C J, Widdershoven G A. Patients as partners in responsive research: methodological notions for collaborations in mixed research teams. Qual Health Res 2009; 19: 401-415. 
19. Viergever R F, Olifson S, Ghaffar A, Terry R F. A checklist for health research priority setting: nine common themes of good practice. Health Res Policy Syst 2010; 8: 36

20. Oral and Dental Health Priority Setting Partnership. Background. Available at https:// oralanddentalhealthpsp.wordpress.com/ (accessed May 2019).

21. Brocklehurst PR, Mackay L, Goldthorpe J, Pretty I A. Older people and oral health: setting a patient-centred research agenda. Gerodontology 2015; 32: 222-228.

22. Everaars B, Jerković́-Ćosić K, van der Putten G J, van der Heijden G J M G. Probing problems and priorities in oral health (care) among community dwelling elderly in the Netherlands a mixed method study. Int J Health Sci Res 2015; 5: 415-429.

23. Everaars $B$, Jerkovicć-Ćosić $K$, van der Putten G J, Pretty I A, Brocklehurst P. Needs in Service Provision for Oral Health Care in Older People: A Comparison Between Greater Manchester (United Kingdom) and Utrecht (the Netherlands). Int J Health Serv 2018; 48: 663-684.

24. Chamberlain P, Wolstenholme D, Dexter M, Seals E. The State of the Art of Design in Health: An expert-led review of the extent of the art of design theory and practice in health and social care. Sheffield: Sheffield Hallam University, 2015.

25. Cooke J, Langley J, Wolstenholme D, Hampshaw S. "Seeing" the Difference: The Importance of Visibility and Action as a Mark of "Authenticity" in Co-production Comment on "Collaboration and Co-production of Knowledge in Healthcare: Opportunities and Challenges". Int J Health Policy Manag 2016; 6: 345-348.
26. Björgvinsson $E$, Ehn $P$, Hillgren $P$ A. Participatory design and "democratizing innovation". Proceedings of the 11th Biennial Participatory Design Conference 1988; 41-50.

27. Lyons M, Smith C, Boaden E et al. Oral care after stroke: where are we now? Eur Stroke J 2018; 3: 347-354.

28. Lafon A, Pereira B, Dufour T et al. Periodontal disease and stroke: a meta-analysis of cohort studies. Eur J Neurol 2014; 21: 1155-1161.

29. Dai R, Lam O L, Lo E C, Li L S, Wen Y, McGrath C. A systematic review and meta-analysis of clinical, microbiological, and behavioural aspects of oral health among patients with stroke. J Dent 2015; 43: 171-180

30. Cassolato S F, Turnbull R S. Xerostomia: clinical aspects and treatment. Gerodontology 2003; 20: 64-77.

31. Sellars C, Bowie L, Bagg J et al. Risk factors for chest infection in acute stroke: a prospective cohort study. Stroke 2007; 38: 2284-2291.

32. Bate P, Robert G. Bringing User Experience to Healthcare Improvement: the concepts, methods and practices of experience-based design. Oxford: Radcliffe Publishing, 2007.

33. Gage M. Making Emotional Connections Through Participatory Design. 2002. Available at http:// boxesandarrows.com/making-emotional-connectionsthrough-participatory-design/ (accessed June 2019).

34. Michie S, Atkins L, West R. The Behaviour Change Wheel: A Guide to Designing Interventions. Sutton: Silverback Publishing, 2014

35. Kirkham J J, Gorst S, Altman D G et al. Core Outcome Set-STAndards for Reporting: The COS-STAR Statement. PLoS Med 2016; 13: e1002148.
36. Mokkink L B, Terwee C B, Patrick D L et al. The COSMIN checklist for assessing the methodological quality of studies on measurement properties of health status measurement instruments: an international Delphi study. Qual Life Res 2010; 19: 539-549.

37. Comet Initiative. Development of a core outcome set for oral health services research involving dependent older adults. 2018. Available at http://www.comet-initiative. org/studies/details/1081?result=true (accessed June 2019).

38. Brocklehurst $P$, Williams $L$, Hoare $Z$ et al. Strategies to prevent oral disease in dependent older people. Cochrane Database Syst Rev 2016; DOI: 10.1002/14651858.CD012402

39. Taylor J, Walsh T, Worthington $\mathrm{H}$, Brocklehurst $\mathrm{P}$, Pemberton M N, Glenny A M. Cochrane and the COMET initiative: developing the evidence base in oral medicine. Br Dent J 2017; 223: 729-732.

40. Williamson P R, Altman D G, Blazeby J M et al. Developing core outcome sets for clinical trials: issues to consider. Trials 2012; 13: 132.

41. de Wit M, Abma T, Koelewijn-van Loon M, Collins S, Kirwan J. Involving patient research partners has a significant impact on outcomes research: a responsive evaluation of the international OMERACT conferences. BMJ Open 2013; 3: e002241.

42. Kirkham J J, Clarke M, Williamson PR. A methodological approach for assessing the uptake of core outcome sets using ClinicalTrials.gov: findings from a review of randomised controlled trials of rheumatoid arthritis. $B M$ 2017; 357: j2262. 